\title{
A NOTE ON TOPOLOGICAL DYNAMICS AND LIMITING EQUATIONS
}

\section{S. M. SHAMIM IMDADI AND M. RAMA MOHANA RAO}

Abstract. A theorem on uniform asymptotic stability of the null solution of a system of differential equations is proved while assuming that the null solution of a limiting equation is uniformly asymptotically stable. This generalizes some of the results of L. Markus.

1. The concept of the set of limiting equations of a given differential equation has been introduced by G. R. Sell [4]. The notion of asymptotically autonomous differential equations introduced by $\mathrm{L}$. Markus [2] can be described as those differential equations for which the set of limiting equations consists of a single point. In [4], G. R. Sell has proved a theorem on asymptotic stability of the null solution of a given differential equation while assuming that the null solution of the given differential equation is uniformly stable and the null solution of every limiting equation is asymptotically stable (in a uniform sense). However, as pointed out in a remark $([4$, p. 273] and $[5$, p. 536]), his theorem does not generalize a result of L. Markus [2, Theorem 2]. The aim of this paper is to prove a theorem on uniform asymptotic stability which generalizes the result of $\mathrm{L}$. Markus [2, Theorem 2].

2. Throughout this paper, we follow the same notation as in [3] and [4]. Let $W$ be an open set in $R^{n}$, Euclidean $n$-space. The Euclidean norm on $R^{n}$ will be denoted by $|x|$. Let $C=C\left(W \times R, R^{n}\right)$ denote the set of all continuous functions $f$ defined on $W \times R$ with values in $R^{n}$. We shall say that a function $f$ is admissible [3] if (i) $f \in C$, and (ii) the solutions of the differential equation $x^{\prime}=f(x, t)$ are unique. By the second condition we mean that given any point $\left(x_{0}, t_{0}\right)$ in $W \times R$, there is precisely one solution $\phi$ of $x^{\prime}=f(x, t)$ that satisfies $\phi\left(t_{0}\right)=x_{0}$. It is evident that if $f$ is an admissible function, then every translate $f_{\tau}$ of $f$ (where $f_{r}(x, t)=f(x, t+\tau)$ ) is an admissible function. Also, if $f$ satisfies the global existence property, then so does each $f_{\tau}$. Let $F=\left\{f_{\tau}: \tau \in R\right\}$ be the space of translates of $f$; then $F$ is a

Received by the editors September 25, 1972 and, in revised form, November 7, 1972 and January 2, 1973.

AMS (MOS) subject classifications (1970). Primary 34C35.

Key words and phrases. Limiting equation, admissible function, regular function.

(c) American Mathematical Society 1973 
subset of $C$. Now let $f$ be an admissible function and consider the space of translates $F$ in the compact open topology [1] on $C$. Let $F_{\mathrm{co}}^{*}=\mathrm{Cl} F$ (that is, the closure in the compact open topology) be the hull of $f$. We shall say that $f$ is regular [3] if every function $f^{*}$ in the hull $F_{\mathrm{co}}^{*}$ is admissible. It is shown in [3] that the mapping $\pi^{*}: F_{\mathrm{co}}^{*} \times R \rightarrow F_{\mathrm{co}}^{*}$, defined by $\pi^{*}\left(f^{*}, t\right)=f_{t}^{*}$, is a dynamical system on $F_{\mathrm{co}}^{*}$, when $F_{\mathrm{co}}^{*}$ has the compact open topology. We shall say that the motion $f_{t}$ is positively compact [3] if the closure of $\left\{\pi^{*}(f, t): t \geqq 0\right\}$ lies in a compact subset of $F_{\mathrm{co}}^{*}$.

Definition of Limiting equations [4]. Let $f \in C$ and let $F_{\mathrm{co}}^{*}$ be the hull of $f$ (neither regularity nor admissibility of $f$ will be important here). Let $\pi^{*}(f, t)=f_{t}$ be the flow on $F_{\mathrm{co}}^{*}$ and let $\Omega_{f}^{*}$ denote the $\omega$-limit set of $f$ in this flow. If the $\omega$-limit set $\Omega_{f}^{*}$ of $f$ in $F_{\mathrm{co}}^{*}$ is nonempty, then we say that the set of limiting equations for

$$
x^{\prime}=f(x, t)
$$

is the set of all differential equations of the form

$$
x^{\prime}=f^{*}(x, t),
$$

where $f^{*} \in \Omega_{f}^{*}$.

We need the following result in our subsequent discussion.

LEMMA 2.1 (KAMKE). Let $\left\{g_{n}\right\}$ be a sequence of continuous functions in $C$, and let $g=\lim g_{n}$, where the convergence is in the compact open topology on $C$. Let $\phi_{n}$ be a solution of $x^{\prime}=g_{n}(x, t)$ with $\phi_{n}(0) \rightarrow x_{0} \in W$. Then there is a subsequence of $\left\{\phi_{n}\right\}$ that converges to a solution $\phi$ of $x^{\prime}=g(x, t)$ that satisfies $\phi(0)=x_{0}$, and the convergence is uniform on compact sets in the interval of definition of $\phi$. If, in addition, the solutions of $x^{\prime}=g(x, t)$ are unique, then $\phi=\lim \phi_{n}$, where the convergence is uniform on compact sets in the interval of definition of $\phi$.

In Theorems 4 and 5 of [4], G. R. Sell has proved that if the equation (2.1), with the assumption that $f$ is a regular function and $f(0, t)=0$ for all $t \geqq 0$, has a "stable" solution, then the limiting equations $(2.2)$ have the same property. The problem of reversing these roles is a bit delicate. That is, if we assume some stability properties of the solutions of (2.2), then it is generally harder to derive results about the given equation (2.1). However, he proved the following result.

THEOREM 2.1 [4, THEOREM 6]. Let $f \in C$ be a regular function with $f(0, t)=0(t \geqq 0)$ and assume that the motion $f_{t}$ is positively compact, in the compact open topology. If (i) the null solution of (2.1) is uniformly stable, and (ii) the null solution of every limiting equation (2.2) is asymptotically stable (in a uniform sense), that is $\left|\phi\left(x_{0}, f^{*}, t\right)\right| \rightarrow 0$ as $t \rightarrow \infty$ whenever 
$\left|x_{0}\right| \leqq a$ and $f^{*} \in \Omega_{f}^{*}$, then the null solution of the given equation (2.1) is asymptotically stable.

3. Now, we shall prove the following main result.

THEOREM 3.1. Let $f$ be a regular function with $f(0, t)=0(t \geqq 0)$. If there exists a function $f^{*}$ in $\Omega_{f}^{*}$ such that the null solution of (2.2) is uniformly asymptotically stable, then the null solution of the given equation (2.1) is uniformly asymptotically stable.

Proof. Let $\phi\left(x_{0}, f^{*}, t\right)$ be any solution of (2.2) with $\phi\left(x_{0}, f^{*}, 0\right)=x_{0}$. Let $\varepsilon>0$ be given. Since the null solution of (2.2) is uniformly asymptotically stable, given $\varepsilon / 2$ there exists a $\delta_{1}=\delta_{1}(\varepsilon / 2)>0$ (without any loss of generality we can suppose that $\left.\delta_{1}<\varepsilon / 2\right)$ such that the inequality $\left|x_{0}\right|<\delta_{1}$ implies

$$
\left|\phi\left(x_{0}, f^{*}, t\right)\right|<\varepsilon / 2 \quad \text { for } t \geqq 0,
$$

and there exists a $\delta_{0}>0$, such that for every $\eta>0$ there exists a $T=T(\eta)>0$ such that the inequality $\left|x_{0}\right|<\delta_{0}$ implies that

$$
\left|\phi\left(x_{0}, f^{*}, t\right)\right|<\eta \quad \text { for } t \geqq T .
$$

Choose $\delta=\min \left(\delta_{1}, \delta_{0}\right)$. From (3.1) and (3.2), we have

$$
\left|\phi\left(x_{0}, f^{*}, t\right)\right|<\varepsilon / 2 \quad \text { for } t \geqq 0,
$$

whenever $\left|x_{0}\right|<\delta$, and for $\delta / 2$ there exists a $T=T(\delta / 2)$ such that the inequality $\left|x_{0}\right|<\delta$ implies

$$
\left|\phi\left(x_{0}, f^{*}, t\right)\right|<\delta / 2 \text { for } t \geqq T .
$$

Now, by Lemma 2.1, given $f^{*} \in \Omega_{f}^{*}$ and $\delta / 2$ there is a $d=d(\delta / 2)>0$ such that

$$
\left|\phi\left(x_{0}, f^{*}, t\right)-\phi\left(x_{0}, g, t\right)\right|<\delta / 2, \quad t \in[0, T],
$$

$\left|x_{0}\right|<\delta$, whenever $\rho\left(f^{*}, g\right)<d$, where $\rho$ is any metric which generates the compact open topology on $F_{\mathrm{co}}^{*}$. By the definition of $\Omega_{f}^{*}$ we can find a translate $f_{\tau}, \tau \geqq 0$, such that $\rho\left(f^{*}, f_{\tau}\right)<d$. Therefore

$$
\left|\phi\left(x_{0}, f^{*}, t\right)-\phi\left(x_{0}, f_{\tau}, t\right)\right|<\delta / 2 \text { for } t \in[0, T],\left|x_{0}\right|<\delta .
$$

Combining (3.3) and (3.5), we get

$$
\begin{aligned}
\left|\phi\left(x_{0}, f_{r}, t\right)\right| & \leqq\left|\phi\left(x_{0}, f^{*}, t\right)\right|+\left|\phi\left(x_{0}, f_{r}, t\right)-\phi\left(x_{0}, f^{*}, t\right)\right| \\
& <\varepsilon / 2+\delta / 2<\varepsilon / 2+\varepsilon / 2=\varepsilon .
\end{aligned}
$$

That is, $\left|\phi\left(x_{0}, f_{\tau}, t\right)\right|<\varepsilon$ for $t \in[0, T],\left|x_{0}\right|<\delta$. Now, let $x_{1}=\phi\left(x_{0}, f_{r}, T\right)$; then from (3.4) and (3.5) we have $\left|x_{1}\right| \leqq\left|\phi\left(x_{0}, f^{*}, T\right)\right|+\left|x_{1}-\phi\left(x_{0}, f^{*}, T\right)\right|<$ $\delta / 2+\delta / 2=\delta$. Therefore, from (3.6) it follows that $\left|\phi\left(x_{1}, f_{r}, t\right)\right|<\varepsilon$ for 
$t \in[0, T]$, which in turn implies that $\left|\phi\left(x_{0}, f_{\tau}, t\right)\right|<\varepsilon$ for $t \in[0,2 T]$, $\left|x_{0}\right|<\delta$. Similarly, if we suppose $x_{2}=\phi\left(x_{1}, f_{r}, T\right)$, then $\left|x_{2}\right| \leqq\left|\phi\left(x_{1}, f^{*}, T\right)\right|+$ $\left|x_{2}-\phi\left(x_{1}, f^{*}, T\right)\right|<\delta / 2+\delta / 2=\delta$, and again, from (3.6), it follows that $\left|\phi\left(x_{0}, f_{\tau}, t\right)\right|<\varepsilon$ for $t \in[0,3 T],\left|x_{0}\right|<\delta$. Now, let $m$ be a positive integer and assumie that $\left|\phi\left(x_{0}, f_{\tau}, t\right)\right|<\varepsilon$ for $t \in[0, m T],\left|x_{0}\right|<\delta$, and assume that $\left|\phi\left(x_{0}, f_{\tau}, m T\right)\right|<\delta$. Let $x_{m}=\phi\left(x_{0}, f_{r}, m T\right)$; then from (3.3) and (3.5), we have

$$
\begin{aligned}
\left|\phi\left(x_{m}, f_{\tau}, t\right)\right| & \leqq\left|\phi\left(x_{m}, f^{*}, t\right)\right|+\left|\phi\left(x_{m}, f_{r}, t\right)-\phi\left(x_{m}, f^{*}, t\right)\right| \\
& <\varepsilon / 2+\delta / 2<\varepsilon / 2+\varepsilon / 2=\varepsilon,
\end{aligned}
$$

for $t \in[0, T]$. Therefore, $\phi\left(x_{0}, f_{\tau}, t\right)$ can be continued to the interval $[m T,(m+1) T]$ on which $\left|\phi\left(x_{0}, f_{\tau}, t\right)\right|<\varepsilon$. Let $x_{m+1}=\phi\left(x_{0}, f_{\tau},(m+1) T\right)$; then from (3.4) and (3.5) and the fact that $\left|x_{m}\right|<\delta$, we have $\left|x_{m+1}\right| \leqq$ $\left|\phi\left(x_{m}, f^{*}, T\right)\right|+\left|x_{m+1}-\phi\left(x_{m}, f^{*}, T\right)\right|<\delta / 2+\delta / 2=\delta$. Thus, by induction, $\left|\phi\left(x_{0}, f_{\tau}, t\right)\right|<\varepsilon$, whenever $\left|x_{0}\right|<\delta$, on every interval $[m T,(m+1) T]$ and hence on $[0, \infty)$. Since $\delta$ is independent of $\tau$, we have, for a given $\varepsilon>0$ there exist a $\delta=\delta(\varepsilon)>0$ and a $\tau=\tau(\varepsilon) \geqq 0$ such that $\left|\phi\left(x_{0}, f, t\right)\right|<\varepsilon, t \geqq \tau$, provided $\left|x_{0}\right|<\delta$. Now from the continuity of solutions with respect to the initial values and the uniqueness of solutions, it follows that the null solution of (2.1) is uniformly stable. For the rest of the proof, choose $\hat{\delta}_{0}=\delta_{0}$ and fix $\left|x_{0}\right|<\hat{\delta}_{0}$. Let $\eta>0$ be given. Choose $\bar{\delta}(\eta)=\min \left(\delta_{1}(\eta / 2), \hat{\delta}_{0}\right)$, $0<\bar{\delta}<\eta$, and $T_{1}(\eta)=T(\bar{\delta} / 2)$. Let $y_{0}=\phi\left(x_{0}, f_{r}, T_{1}\right), \tau=\tau(\eta) \geqq 0$. Then

$$
\left|y_{0}\right| \leqq\left|\phi\left(x_{0}, f^{*}, T_{1}\right)\right|+\left|\phi\left(x_{0}, f_{r}, T_{1}\right)-\phi\left(x_{0}, f^{*}, T_{1}\right)\right|<\bar{\delta} / 2+\bar{\delta} / 2=\bar{\delta} .
$$

Thus by the first part of the proof, $\left|\phi\left(y_{0}, f, t\right)\right|<\eta$ for $t \geqq \tau$, whenever $\left|y_{0}\right|<\bar{\delta}(\eta)$. Now by the uniqueness of solutions, it follows that $\left|\phi\left(x_{0}, f, t\right)\right|<$ $\eta$ for $t \geqq T^{*}$, whenever $\left|x_{0}\right|<\hat{\delta}_{0}$, where $T^{*}=T^{*}(\eta)=\tau+T_{1}$. This completes the proof of the theorem.

Remark 3.1. Observe that in Theorem 2.1, G. R. Sell assumed that the motion $f_{t}$ is positively compact and the null solution of every limiting equation (2.2) is asymptotically stable (in a uniform sense) to prove that the null solution of (2.1) is asymptotically stable. Clearly, Theorem 2.1 does not generalize a result of L. Markus [2, Theorem 2] as remarked in $[4$, p. 273] and [5, p. 536]. It is appropriate to remark here that, in Theorem 3.1, we merely assume that the null solution of (2.2) for some $f^{*}$ in $\Omega_{f}^{*}$ is uniformly asymptotically stable to prove that the null solution of (2.1) is uniformly asymptotically stable. Obviously, this would generalize the result of L. Markus [2, Theorem 2] for asymptotically autonomous equations.

The authors wish to thank the referee for his valuable suggestions. 


\section{REFERENCES}

1. J. L. Kelley, General topology, Van Nostrand, Princeton, N.J., 1955. MR 16, 1136.

2. L. Markus, Asymptotically autonomous differential systems, Contributions to Nonlinear Oscillations, Vol. 3, Ann. of Math. Studies, no. 36, Princeton Univ. Press, Princeton, N.J., 1956, pp. 17-29. MR 18, 394.

3. G. R. Sell, Non-autonomous differential equations and topological dynamics. I. The basic theory, Trans. Amer. Math. Soc. 127 (1967), 241-262. MR 35 \#3187a.

4. - Non-autonomous differential equations and topological dynamics. II. Limiting equations, Trans. Amer. Math. Soc. 127 (1967), 263-283. MR 35 \#3187b.

5. - Non-autonomous differential equations as dynamical systems, Proc. Internat. Sympos. Differential Equations and Dynamical Systems (Puerto Rico, 1965), Academic Press, New York, 1967, pp. 531-536.

Department of Mathematics, Indian Institute of Technology, Kanpur 16, INDIA 